\title{
Prior transient ischemic attacks may have a neuroprotective effect in patients with ischemic stroke
}

\author{
Wei-Wei Wang, De-Zhe Chen, Min Zhao, Xia-Feng Yang, Dian-Rong Gong
}

Department of Neurology, LiaoCheng Hospital, Liaocheng, China

Submitted: 26 May 2016

Accepted: 5 August 2016

Arch Med Sci 2017; 13, 5: 1057-1061

DOI: 10.5114 /aoms.2016.63744

Copyright @ 2016 Termedia \& Banach

\section{Abstract}

Introduction: Although functional recovery and survival after ischemic infarction seem to improve in patients with prior transient ischemic attack (TIA), little is known about the role of characteristics of prior TIA in subsequent cerebral infarction. Thus, the objective of this study was to explore how the characteristics of prior TIA have a neuroprotective effect on patients with ischemic stroke.

Material and methods: A total of 221 patients admitted consecutively to a primary care center for first-ever ischemic stroke were divided into two groups on the basis of the presence or absence of prior TIAs. The initial NIHSS modified Rankin Scale was used to measure the severity and disability after the stroke. Subgroups were based on the TIA duration ( $<10 \mathrm{~min}, 10$ to $60 \mathrm{~min}$, and $>60 \mathrm{~min}$ ), TIA frequency (1 time, 2-3 times, more than 3 times), and the interval of stroke ( $<1$ week, $1-4$ weeks, $>4$ weeks). The severity of the neurologic picture on admission and functional disability after stroke were compared between patients with and without TIAs and subgroups as well.

Results: A total of 132 (59.73\%) of the 221 patients had prior TIAs before stroke. Risk factors and the initial clinical picture did not differ between patients with or without TIAs. Patients with prior TIA had a more favorable outcome than those without TIA (59.09\% vs. $43.82 \%)$, and a significant difference between the two groups was observed $\left(\chi^{2}=4.976, p=0.026\right)$. Furthermore, neurological outcome in patients with prior TIA lasting for $60 \mathrm{~min}$, less than 3 times and shorter intervals within 4 weeks was significantly different from that in the non-TIA group $(p<0.05)$.

Conclusions: Prior transient ischemic attacks may have a neuroprotective effect on the subsequent ischemic stroke, and this effect might be affected by the characteristics of TIAs. Patients with TIAs of low frequency, short duration and short interval are considered to have better neurological outcomes.

Key words: transient ischemic attack, ischemic stroke, neuroprotection, ischemic tolerance.

\section{Introduction}

It is well established that transient ischemic attacks (TIA) increase the risk of ischemic stroke $[1,2]$. Transient ischemic attacks occur not long ( $<1$ month) before ischemic stroke in $15 \%$ of patients, and most patients recover within $1 \mathrm{~h}[1,2]$, and normally, TIAs last no longer than $15 \mathrm{~min}$ [3]. There are no differences in initial stroke severity in cerebral infarction $(\mathrm{Cl})$ patients with or without prior TIAs. Some experimental findings indicate the opposite view. Animal models of ischemic stroke have

\author{
Corresponding author: \\ De-Zhe Chen \\ Department of Neurology \\ LiaoCheng Hospital \\ No. 45 Huashan Rd \\ Kaifa District \\ 252000 Liaocheng, China \\ Phone: +860635851746 \\ E-mail: chen_dezhe@126.com
}


recently provided evidence for the induction of a phenomenon of "ischemic tolerance" in rodents at advanced age subjected to short ischemic episodes, rendering the brain more tolerant to subsequent persistent cerebral ischemia [4-6]. This effect was initially observed in the CA1 zone of the hippocampus [7], followed by other subfields in the hippocampus and in the frontoparietal cortex, thalamus, and basal ganglia [8].

Furthermore, more and more clinical studies demonstrate that patients with $\mathrm{Cl}$ in the anterior circulation who have had a prior TIA, triggering the mechanisms involved in the "focal ischemic tolerance phenomenon", present a less severe neurologic picture and, consequently, a better prognosis than patients with ischemic stroke in the anterior circulation who have not had a previous ipsilateral TIA [9]. However, there are few reports regarding the characteristics of TIA following ischemic stroke. We analyzed the outcome of 2 groups of patients with cerebral infarction according to the presence or absence of prior TIA. A separate analysis of the clinical features of the TIA was also performed.

\section{Material and methods}

The present study sample consisted of all consecutive patients with first-ever ischemic stroke (Liaocheng People's Hospital). The study was approved by the local ethics committees. We retrospectively reviewed 221 cases, from 2012 to 2014 , of patients with a diagnosis of acute stroke with or without prior TIA. The inclusion criteria were the Chinese diagnosis guideline for TIA [4] and acute ischaemic stroke [5]. Exclusion criteria were cerebral embolism, cerebral ischaemia from causes other than atherosclerosis, such as intracranial arteritis and CADASIL, hemorrhagic stroke or subarachnoid hemorrhage, TIA in a vascular territory other than that of the subsequent stroke and epilepsy. Two hundred and fifteen patients fulfilled the criteria and were divided into two groups based on the presence of stroke with or without prior TIA. Patients were treated with aspirin and clopidogrel separately or with a combination of the two. All of the patients were hospitalized and received similar medical treatments and rehabilitation. The history of previous TIAs was recorded from patients' medical history.

Of the patients presenting with stroke with prior TIA, 120 (90.91\%) were treated with aspirin and 52 (39.39\%) were treated with clopidogrel. Of the patients presenting with stroke without prior TIA, 78 (87.64\%) were treated with aspirin and 20 (33.71\%) patients were treated with clopidogrel. In more detail, 63 patients/132 (47.73\%) received aspirin in the group of stroke with previous TIA before stroke because of the previous TIA and 36 patients/89 (40.45\%) had the aspirin medication in the group of stroke without TIA attacks because of the existing risk factors for stroke.

Clinical characteristics included gender, age, incidence of hypertension (HTN), diabetes mellitus (DM), smoking, drinking, dyslipidemia, atrial fibrillation (AF), myocardial infarction (MI), peripheral vascular disease (PVD) and initial National Institutes of Health Stroke Scale (NIHSS) score. The NIHSS evaluation was performed upon admis sion. The neurological outcome at three months post-onset was divided based on the modified Rankin Scale (mRS) into good outcome with 0-1 and poor outcome with 2-6.

\section{Statistical analysis}

Baseline measurements and cardiovascular risk factors are reported as mean $\pm \mathrm{SD}$. The $t$-test was used to evaluate differences between the two groups. Initial NIHSS and chronic mRS were evaluated by nonparametric statistics due to the same sample size and non-normal distributions. Thus, differences between groups were evaluated using Mann-Whitney tests. A $\chi^{2}$ test was used to analyze the enumeration data. A $p$-value of $<0.05$ was considered statistically significant. Data were analyzed using SPSS v11.0 software.

\section{Results}

The two groups were comparable with respect to demographic characteristics and cardiovascular risk factors. In our study, comparison of the initial NIHSS scores between the two groups revealed no significant differences (Table I; $p>0.05$ ). The time from admission to discharge was also comparable in both groups, and therefore mRS difference could serve as a reasonable parameter for considering the neurological outcome of subsequent stroke attack. The mean duration of the TIA was $42.68 \pm 11.23 \mathrm{~s}$, mean frequency was $2.14 \pm 0.45$, and mean interval between TIA and stroke was $2.63 \pm 0.72$ weeks.

In our study, comparison of the neurological outcome by mRS between the two groups revealed a significant difference (Table II). Eightytwo $(62.12 \%)$ stroke patients with prior TIAs had a good outcome, while only 39 (43.82\%) stroke patients without prior TIAs had a good outcome $\left(\chi^{2}=4.834, p=0.0089\right)$.

As we showed that the prior TIAs had a neuroprotective effect on subsequent ischemic stroke, we further investigated whether an association exists between characteristics of prior TIA and stroke outcome. Here, we mostly focused on the special groups of TIA with duration, different frequency and TIA-stroke interval. For each subdi- 
Table I. Comparison of characteristics between two groups

\begin{tabular}{|lccc|}
\hline Characteristic & $\begin{array}{c}\text { Stroke with prior TIA } \\
(N=132)\end{array}$ & $\begin{array}{c}\text { Stroke without prior TIA } \\
(\boldsymbol{N}=89)\end{array}$ & $P$-value \\
\hline Male/female & $75 / 57$ & $51 / 38$ & 0.50 \\
\hline Age [years] & $62.3 \pm 10.6$ & $59.8 \pm 9.6$ & 0.32 \\
\hline HTN, $n$ (\%) & $80(60.61)$ & $55(61.80)$ & 0.89 \\
\hline DM, $n$ (\%) & $66(50.00)$ & $43(48.31)$ & 0.89 \\
\hline Smoking, $n(\%)$ & $82(62.12)$ & $51(57.30)$ & 0.11 \\
\hline Drinking, $n$ (\%) & $56(42.42)$ & $47(52.81)$ & 0.13 \\
\hline Dyslipidemia, $n$ (\%) & $44(33.33)$ & $29(32.58)$ & 1.00 \\
\hline AF, $n$ (\%) & $12(9.09)$ & $6(6.74)$ & 0.62 \\
\hline MI, $n$ (\%) & $32(24.24)$ & $23(25.84)$ & 0.87 \\
\hline PVD, $n$ (\%) & $8(6.06)$ & $5(8.99)$ & 1.00 \\
\hline Anticoagulant or antiplatelet, $n(\%)$ & $112(92.42)$ & $73(82.02)$ & 1.00 \\
\hline Lipid-lowering medication, $n$ (\%) & $42(31.82)$ & $24(26.97)$ & 1.00 \\
\hline DSA intervention, $n$ (\%) & $12(9.09)$ & $3(3.37)$ & 0.77 \\
\hline Initial NIHSS & $15 \pm 3.8$ & $17 \pm 5.2$ & 0.93 \\
\hline
\end{tabular}

DSA - digital subtraction angiography.

vision, we conducted a separate analysis of the $\mathrm{mRS}$ scores relative to those from patients with no prior TIA.

As for the TIA duration (Table III), the data from patients with prior TIAs were further subdivided into (1) TIA lasting for $10 \mathrm{~min}$; (2) 10-60 min; and (3) more than $60 \mathrm{~min}$. We found that only those stroke patients with TIA 60 min or less developed better neurological outcomes compared to the respective groups $(p<0.05)$. There was no significant difference in stroke patients with TIA more than $60 \min (p>0.05)$.

Regarding the TIA frequency (Table IV), patients with prior TIAs were further divided into (1) one-time TIA; (2) 2-3 times TIA; and (3) > 3 times TIAs. We observed that stroke patients with
Table II. Neurological outcomes between two groups

\begin{tabular}{|lcc|}
\hline Group & Good outcome & Poor outcome \\
\hline $\begin{array}{l}\text { Stroke with TIA } \\
(n=132)\end{array}$ & $82(62.12 \%)$ & $50(37.88 \%)$ \\
\hline $\begin{array}{l}\text { Stroke without TIA } \\
(n=89)\end{array}$ & $39(43.82 \%)$ & $50(56.18 \%)$ \\
\hline$\chi^{2}$ & 4.834 \\
\hline$P$-value & 0.0089 \\
\hline
\end{tabular}

prior TIAs up to three times had better neurological outcomes compared to the respective groups $(p<0.05)$. Those stroke patients with more than three TIAs before the onset did not demonstrate a beyond neurological outcome $(p>0.05)$.

Table III. Duration of TIA and subsequent ischemic stroke

\begin{tabular}{|lcccc|}
\hline Group & $\begin{array}{c}\text { Total without prior TIA } \\
(n=89)\end{array}$ & $\begin{array}{c}<10 \mathrm{~min} \\
(n=37)\end{array}$ & $\begin{array}{c}10-60 \mathrm{~min} \\
(n=42)\end{array}$ & $\begin{array}{c}>60 \mathrm{~min} \\
(n=53)\end{array}$ \\
\hline Good outcome & $39(43.82 \%)$ & $30(81.08 \%)$ & $29(59.52 \%)$ & $23(43.40 \%)$ \\
\hline Poor outcome & $50(56.18 \%)$ & $7(18.92 \%)$ & $13(40.48 \%)$ & $30(56.60 \%)$ \\
\hline$P$-value & & 0.0001 & 0.0070 & 0.9607 \\
\hline
\end{tabular}

Table IV. Frequency of prior TIA and subsequent stroke

\begin{tabular}{|lcccc|}
\hline Group & $\begin{array}{c}\text { Total without prior TIA } \\
(n=89)\end{array}$ & $\begin{array}{c}\text { Once } \\
(n=45)\end{array}$ & $\begin{array}{c}\text { 2-3 times } \\
(n=42)\end{array}$ & $\begin{array}{c}>3 \text { times } \\
(n=45)\end{array}$ \\
\hline Good outcome & $39(43.82 \%)$ & $30(66.67 \%)$ & $27(64.29 \%)$ & $25(55.56 \%)$ \\
\hline Poor outcome & $50(56.18 \%)$ & $15(33.33 \%)$ & $15(35.71 \%)$ & $20(44.44 \%)$ \\
\hline$P$-value & & 0.0171 & 0.0392 & 0.1990 \\
\hline
\end{tabular}


Table V. TIA-stroke interval and subsequent stroke

\begin{tabular}{|lcccc|}
\hline Group & $\begin{array}{c}\text { Total without prior TIA } \\
(n=89)\end{array}$ & $<1$ week $(n=29)$ & 1-4 weeks $(n=34)$ & $>4$ weeks $(n=69)$ \\
\hline Good outcome & $39(43.82 \%)$ & $24(82.76 \%)$ & $22(64.71 \%)$ & $36(52.17 \%)$ \\
\hline Poor outcome & $50(56.18 \%)$ & $5(17.24 \%)$ & $12(35.29 \%)$ & $33(47.83 \%)$ \\
\hline$P$-value & & 0.0003 & 0.0383 & 0.3368 \\
\hline
\end{tabular}

When it comes to the TIA-stroke interval (Table V), patients were grouped into (1) within 1 week; (2) 1-4 weeks; and (3) more than 4 weeks. Those patients with the TIA-stroke interval less than 4 weeks showed better neurological outcomes $(p<0.05)$. Also there was no significant difference regarding patients with a TIA-stroke interval more than 4 weeks $(p>0.05)$.

\section{Discussion}

In this study, we found that ischemic stroke patients with prior TIAs had a better neurological outcome compared to those without prior TIAs $(p<0.05)$. In addition, the subgroup analysis demonstrated that the characteristics of TIAs had an effect on the neuroprotection of prior TIAs. Stroke patients with TIAs of 60 min or more had a poorer neurological outcome compared to those with TIAs of less than $60 \mathrm{~min}$, while stroke patients with more than 3 times prior TIAs also had a poorer outcome as well. Patients with more severe prior TIAs with longer duration and higher frequencies had a poorer outcome. This means that after TIAs, the brain still undergoes a period of hypoxia and ischemia; however, if this hypoxic period extends much longer, this neuroprotective effect would be replaced by serious brain injury $[4,10]$. When the interval between the TIA and stroke became longer, these patients had a poorer outcome as well, indicating that the neuroprotection of prior TIAs had a window effect. Therefore, although the prior TIAs had a neuroprotective effect on subsequent cerebral ischemia, this effect would be affected by the characteristics of prior TIAs including the duration, frequency and interval between TIA and stroke. The mechanism of this effect needs further studies.

The findings from our study are relatively consistent with previous reports. One study found that prior TIA occurs mostly within 7 days [11, 12] or 1 month before the onset of ischemic stroke [9]. Moncayo et al. reported that those patients with TIA less than 30 min had a better neurological outcome compared to those with TIAs longer than $30 \mathrm{~min}$ [9]. They considered that those patients with prior TIAs less than 30 min had a better capacity of brain resistance to hypoxia and ischemia. Arboix et al. [13] and Sitzer et al. [14] found that in patients with non-lacunar infarction, patients with- out TIAs had a poorer neurological outcome than those with TIAs. This is further confirmed by magnetic resonance imaging data showing a smaller infarction area in stroke patients with prior TIAs [15].

The mechanism underlying how prior TIA improved the outcome of subsequent stroke might be related to the capacity for resistance to the ischemia due to atherosclerosis. However, this effect is compromised in patients with lacunar infarction $[9,16]$. From the pathophysiological point of view, our results might be related to the neuroprotection induced by ischemic tolerance [8]. Actually, several animal models of brain ischemia have proved that prior hypoxia can improve the neurological outcome after cerebrovascular events via endogenous neuroprotection, including the activating transcription factor and post-translational modification [10, 17]. To further explore the molecular mechanism underlying these observations, it would be beneficial to look for a new prophylactic strategy for ischemic stroke.

There are also some limitations of this study. First, there are other possible explanations for the results. The presence of a prior TIA should increase the patient's and medical staff's awareness of the preventive strategy for stroke [18]. This subjective awareness might explain the better neurological outcome in patients with prior TIAs. Second, the information about TIA was received from the patient or family, without any medical track record. Third, some patients take both antiplatelet therapies due to the previous histories including myocardial infarction, TIAs and risk factors related to stroke including hypertension, hyperlipidemia and so on. It would be necessary to stratify patients with different combinations of antiplatelet therapy based on a larger patient sample, to clarify the effects of anticoagulation therapy on the outcome of TIA patients.

In conclusion, our results indicate that prior TIAs may have a neuroprotective effect on the neurological outcome of subsequent ischemic stroke, and this effect is further affected by characteristics of TIAs including the duration, frequency and interval between TIA and stroke.

\section{Conflict of interest}

The author declares no conflict of interest. 


\section{References}

1. Janssen AWM, de Leeuw FE, Janssen MCH. Risk factors for ischemic stroke and transient ischemic attack in patients under age 50. J Thromb Thrombolysis 2011; 31: 85-91.

2. Wu CM, McLaughlin K, Lorenzetti DL, et al. Early risk of stroke after transient ischemic attack: a systematic review and meta-analysis. Arch Intern Med 2007; 167: 2417-22.

3. Amort M, Fluri F, Schäfer J, et al. Transient ischemic attack versus transient ischemic attack mimics: frequency, clinical characteristics and outcome. Cerebrovasc Dis 2011; 32: 57-64.

4. Ledvényiová-Farkašová V, Bernátová I, Balis P, et al. Effect of crowding stress on tolerance to ischemia-reperfusion injury in young male and female hypertensive rats: molecular mechanisms. Can J Physiol Pharmacol 2015; 93: 793-802.

5. Ghanbari M, Jeddi S, Bagheripuor F, Ghasemi A. The effect of maternal hypothyroidism on cardiac function and tolerance to ischemia-reperfusion injury in offspring male and female rats. J Endocrinol Invest 2015; 38: 915-22.

6. Boscia F, Casamassa A, Secondo A, et al. NCX1 exchanger cooperates with calretinin to confer preconditioning-induced tolerance against cerebral ischemia in the striatum. Mol Neurobiol 2015; 1: 1-12.

7. Nasrniya S, Bigdeli MR. Ischemic tolerance induced by normobaric hyperoxia and evaluation of group I and II metabotropic glutamate receptors. Curr Neurovasc Res 2013; 10: 21-8.

8. Kitagawa K, Matsumoto M, Kuwabara K, et al. "Ischemic tolerance" phenomenon detected in various brain regions. Brain Res 1991; 561: 203-11.

9. Moncayo J, de Freitas GR, Bogousslavsky J, et al. Do transient ischemic attacks have a neuroprotective effect? Neurology 2000; 54: 2089-94.

10. Gidday JM. Cerebral preconditioning and ischaemic tolerance. Nat Rev Neurosci 2006; 7: 437-48.

11. Schaller B. Ischemic preconditioning as induction of ischemic tolerance after transient ischemic attacks in human brain: its clinical relevance. Neurosci Lett 2005; 377: 206-11.

12. De Marchis GM, Weck A, Audebert H, et al. Copeptin for the prediction of recurrent cerebrovascular events after transient ischemic attack: results from the CoRisk study. Stroke 2014; 45: 2918-23.

13. Arboix A, Cabeza N, García-Eroles L, et al. Relevance of transient ischemic attack to early neurological recovery after nonlacunar ischemic stroke. Cerebrovasc Dis 2003; 18: 304-11.

14. Sitzer M, Foerch C, Neumann-Haefelin T, et al. Transient ischaemic attack preceding anterior circulation infarction is independently associated with favourable outcome. J Neurol Neurosurg Psychiatry 2004; 75: 659-60.

15. Wegener S, Gottschalk B, Jovanovic V, et al. Transient ischemic attacks before ischemic stroke: preconditioning the human brain? A multicenter magnetic resonance imaging study. Stroke 2004; 35: 616-21.

16. Arboix A, Alió J. Cardioembolic stroke: clinical features, specific cardiac disorders and prognosis. Curr Cardiol Rev 2010; 6: 150-61.

17. Blanco M, Lizasoain I, Sobrino T, et al. Ischemic preconditioning: a novel target for neuroprotective therapy. Cerebrovasc Dis 2005; 21 Suppl 2: 38-47.

18. Karliński M, Gluszkiewicz M, Członkowska A. The accuracy of prehospital diagnosis of acute cerebrovascular accidents: an observational study. Arch Med Sci 2015; 11: 530-5. 